\title{
Reduction of frontal neocortical grey matter associated with affective aggression in patients with temporal lobe epilepsy: an objective voxel by voxel analysis of automatically segmented MRI
}

Friedrich G Woermann, Ludger Tebartz van Elst, Matthias J Koepp, Samantha L Free, Pamela J Thompson, Michael R Trimble, John S Duncan

\begin{abstract}
Background-Interictal episodes of aggression are often reported in patients with epilepsy. Some have characteristics of what has been referred to as episodic dyscontrol or intermittent explosive disorder (IED). Although structural brain abnormalities are thought to play a part in the pathophysiology of aggression, there are few in vivo studies of structural cerebral changes in patients with epilepsy and aggression. Using quantitative MRI, subtle structural brain abnormalities can be investigated in subgroups of patients with both epilepsy and episodes of affective aggression.
\end{abstract}

Methods-After automated segmentation of cerebral grey matter from T1 weighted MRI, the objective technique of statistical parametric mapping (SPM) was applied to the analysis of 35 control subjects, 24 patients with temporal lobe epilepsy (TLE) with a history of repeated, interictal episodes of aggression, and 24 patients with TLE without episodes of aggression. Both TLE patient groups were compared with each other and with the control subjects on a voxel by voxel basis for increases and decreases of grey matter.

Results-The patients with TLE with aggressive episodes had a decrease of grey matter, most markedly in the left frontal lobe, compared with the control group and with patients with TLE without aggressive episodes.

Conclusion-These findings suggest that a reduction of frontal neocortical grey matter might underly the pathophysiology of aggression in TLE. These voxel by voxel comparisons can guide further in vivo studies into aggression.

(F Neurol Neurosurg Psychiatry 2000;68:162-169)

Psychiatry,

Albert-Ludwigs-

Universität, Hauptstr.

5, 79104 Freiburg,

Germany

L T van Elst

Correspondence to: Dr FG Woermann

Received 4 May 1999 and in revised form

7 September 1999

Accepted 17 September 1999 a child, low socioeconomic status, trauma, infection, and minimal brain damage. ${ }^{23}$

Temporolimbic and frontal lobe structures are involved in the neurobiology of aggressive behaviour. ${ }^{4}$ In patients with TLE, we have shown recently, using quantitative MRI, that amygdala or hippocampal sclerosis was not associated with interictal aggressive episodes. ${ }^{5}$ This study also showed lower intellect and higher levels of depression and anxiety in patients with TLE to be associated with interictal episodes of aggression and suggested that more widespread, but subtle, cerebral structural abnormalities were a probable aetiological factor.

We have previously used voxel by voxel group comparisons of structural MRI to show differences in cortical grey matter in different epilepsy syndromes. ${ }^{6}$

The aim of the current study was to investigate the structure of the whole cortical grey matter in patients with TLE with or without episodes of affective aggression, using automated grey matter segmentation of $\mathrm{T} 1$ weighted MRI images and voxel by voxel comparison between these otherwise clinically homogeneous groups of patients with TLE and normal control subjects.

\section{Methods}

CONTROL SUBJECTS AND PATIENTS

Patients with TLE were recruited from a tertiary referral centre (National Society for Epilepsy, National Hospital for Neurology and Neurosurgery). Informed consent was obtained from all patients. A consensus diagnosis of TLE was reached by two neurologists. This diagnosis was based on complex partial seizures with a semiology and EEG findings compatible with a temporal lobe origin in the absence of any EEG or MRI pathology pointing to an extratemporal focus. Patients with an IQ $<70$ or a history of personality disorder or psychoses were not included.

Twenty five patients with TLE with a history of episodic affective aggression (eight women, six left handers; median age 27 years, range 18-49 years) were identified. These patients fulfilled DSM-IV criteria for intermittent explosive disorder (IED; DSM-IV: 312.34) except that they all had an organic brain disease (TLE). ${ }^{7}$ The essential phenomenological feature of IED was the occurrence of discrete episodes of failure to resist aggressive impulses that resulted in serious assaultative acts or 
Table 1 Clinical data in patients with TLE with or without IED

\begin{tabular}{|c|c|c|}
\hline & TLE with IED & TLE without IED \\
\hline Age of onset of epilepsy (median, range; y) & $7(1-28)$ & $8(1-25)$ \\
\hline Duration of epilepsy (median, range; y) & $19(5-45)$ & $23(7-46)$ \\
\hline Seizure frequency (mean/month, (SD)) & $13.4(15.7)$ & $21(38.4)$ \\
\hline SDAS $9(\text { mean, }(\mathrm{SD}))^{\star}$ & $14.9(7.5)$ & $0.4(0.7)$ \\
\hline SDAS $21(\text { mean, }(\mathrm{SD}))^{\star}$ & $30.9(12)$ & $3(4.6)$ \\
\hline FIQ (mean, $(\mathrm{SD}))^{\star}$ & $80.6(8.5)$ & $93(13.9)$ \\
\hline VIQ (mean, $(\mathrm{SD}))^{\star}$ & $81(8.6)$ & $93.8(14.1)$ \\
\hline PIQ (mean, (SD))* & $83.3(11.8)$ & $94.7(15.1)$ \\
\hline $\mathrm{BDI}(\text { mean, }(\mathrm{SD}))^{\star}$ & $8.8(4.8)$ & $4.2(5.8)$ \\
\hline S-STAI (mean, $(\mathrm{SD}))^{\star}$ & $44.1(14.9)$ & $32.1(10.9)$ \\
\hline Verbal fluency (mean, $(\mathrm{SD}))^{\star}$ & $8.3(3.7)$ & $12.2(5.2)$ \\
\hline
\end{tabular}

IED=intermittent explosive disorder; SDAS=social dysfunction and aggression scale; FIQ=full IQ, VIQ=verbal IQ, PIQ=performance IQ, all measured with the HAWIE-R; BDI=Beck's depression inventory; S-STAI $=$ state trait anxiety inventory.

${ }^{\star} \mathrm{p} \leqslant 0.01$.

destruction of property. The degree of aggressivness was out of proportion to any provocation or precipitating psychosocial stressors and was not accounted for by another psychiatric disorder. Twenty five patients with TLE without IED were matched for socioeconomic variables, duration of epilepsy, and seizure severity (10 women, two left handers; median age 33 years, range $19-56$ years). Thirty five healthy volunteers were scanned (17 women; median age 28 years, range $14-55$ years).

The patients with TLE of this current study have been described earlier, using clinical psychiatric and quantitative psychometric assessment as well as visually assessed and rater dependent, volume of interest based quantitative MRI. ${ }^{5}$ Clinical and psychometrical data of the patients with epilepsy are summarised in table 1 . To assess aggression, carers, and next of kin of both TLE patient groups were asked to fill in the social dysfunction and aggression scale (SDAS-21), including a subscale for outward aggression (SDAS-9). ${ }^{8} 9$ Psychopathology was quantified using well established psychometric questionnaires for depression (Beck's depression inventory, BDI) and anxiety (state trait anxiety inventory, STAI). Furthermore all patients received extensive neuropsychological testing including an assessment of overall intelligence. A verbal fluency test was included (produce as many words beginning with the letter $S$ as possible during a period of 1 minute). Diagnoses on visual assessment of MRI are summarised in table 2 .

MRI SCANNING PROTOCOL

Magnetic resonance imaging was performed, using a $1.5 \mathrm{~T}$ GE Signa scanner (Milwaukee, USA). An inversion recovery prepared 3D spoiled gradient echo (IRP-SPGR) sequence (TR/TE/TI/NEX 17.4/4.2/450/1, flip angle 20, matrix size $256 \times 192,24 \times 18 \mathrm{~cm}$ FOV) with 124 contiguous coronal slices and a slice thickness of $1.5 \mathrm{~mm}$ was used for volumetric studies. All MRIs were reviewed by experienced neuroradiologists.

Table 2 MRI diagnosis on visual assessment

\begin{tabular}{|c|c|c|c|c|}
\hline $\begin{array}{l}\text { MRI detected } \\
\text { abnormalities }\end{array}$ & Left temporal & $\begin{array}{l}\text { Right } \\
\text { temporal }\end{array}$ & $\begin{array}{l}\text { Bilateral } \\
\text { temporal }\end{array}$ & No abnormality \\
\hline TLE with IED & $11\left(8 \mathrm{HS}, 3\right.$ others $\left.^{\star}\right)$ & $1 \mathrm{HS}$ & $3 \mathrm{HS}$ & 10 \\
\hline TLE w/o IED & $10 \mathrm{HS}$ & $8 \mathrm{HS}$ & $1 \mathrm{HS}$ & 6 \\
\hline
\end{tabular}

HS=hippocampal sclerosis, ${ }^{\star}$ one with amygdala sclerosis, one with low grade glioma of the amygdala, one with dysembryoblastic neuroepithelial tumour of the amygdala; w/o=without.
DATA ANALYSIS

Data were analysed on a Sun SPARC 20 workstation (Sun Microsystems, Mountain View, CA, USA) using Analyze version 7.5 (Mayo Foundation $^{10}$ ), MATLAB 4.2 a (The MathWorks, Natick, MA, USA), and SPM 96 (Wellcome Department of Cognitive Neurology, Institute of Neurology, London, UK). ${ }^{112}$

ANALYSIS OF STRUCTURAL MRI DATA IN SPM In Analyze, images were first reformatted to the axial plane with voxel dimensions of $0.93 \times 0.93 \times 0.93 \mathrm{~mm}^{3}$. These MR images were spatially normalised, segmented, and smoothed, using SPM 96. On a voxel by voxel basis, automatically segmented grey matter was compared between groups of control subjects and both TLE patient groups. SPM 96 characterises significant regional differences in image parameters, while allowing for global differences to be taken into account, and consists of the following processing steps.

Firstly, the individual T1 weighted MRI volume acquisitions of different brains (the images before segmentation) were transformed into a common standard 3D space without requiring user defined landmarks, using a $\mathrm{T} 1$ weighted MRI template and the default normalisation of SPM 96. Secondly, the SPM segmentation of normalised MRIs employed a clustering algorithm identifying voxel intensities of particular tissue types (grey and white matter, CSF), combined with a priori knowledge about the spatial distribution of these clusters in normal control subjects. ${ }^{13}$ Thirdly, the stereotactically normalised grey matter images were smoothed with a $14 \mathrm{~mm}$ isotropic Gaussian kernel allowing for interindividual gyral variation.

The smoothed grey matter images represented the amount of grey matter per voxel.

To test hypotheses about regionally specific effects in the imaging data, the estimates were compared using two contrasts. This analysis detected whether each voxel had a greater or lesser grey matter density in a patient group, than had the equally localised voxel in the control group or in the other patient group. In addition to these group comparisons between patients and controls, we conducted voxel-wise regression of grey matter density on psychometrical scores describing the whole patient population. To normalise for differences in voxel intensity across scans, global mean voxel value was included as a confounding covariate in an analysis of covariance (ANCOVA). ${ }^{14}$

The resulting set of voxel values for each contrast (more or less grey matter; positive or negative correlation) constitutes a statistical parametric map of the $t$ statistic $\left(\mathrm{SPM}_{\{t}\right)$. The $\mathrm{SPM}_{\{t\}}$ values were transformed to normal distribution and thresholded at $\mathrm{p}<0.001$. To correct for multiple comparisons the resulting foci were characterised in terms of spatial extent $\kappa .{ }^{15}$ This correction describes the probability that a region of the observed size could have occurred by chance over the entire volume analysed (a corrected $p$ value). The corrected $p$ value chosen was $\mathrm{p}<0.05$. 

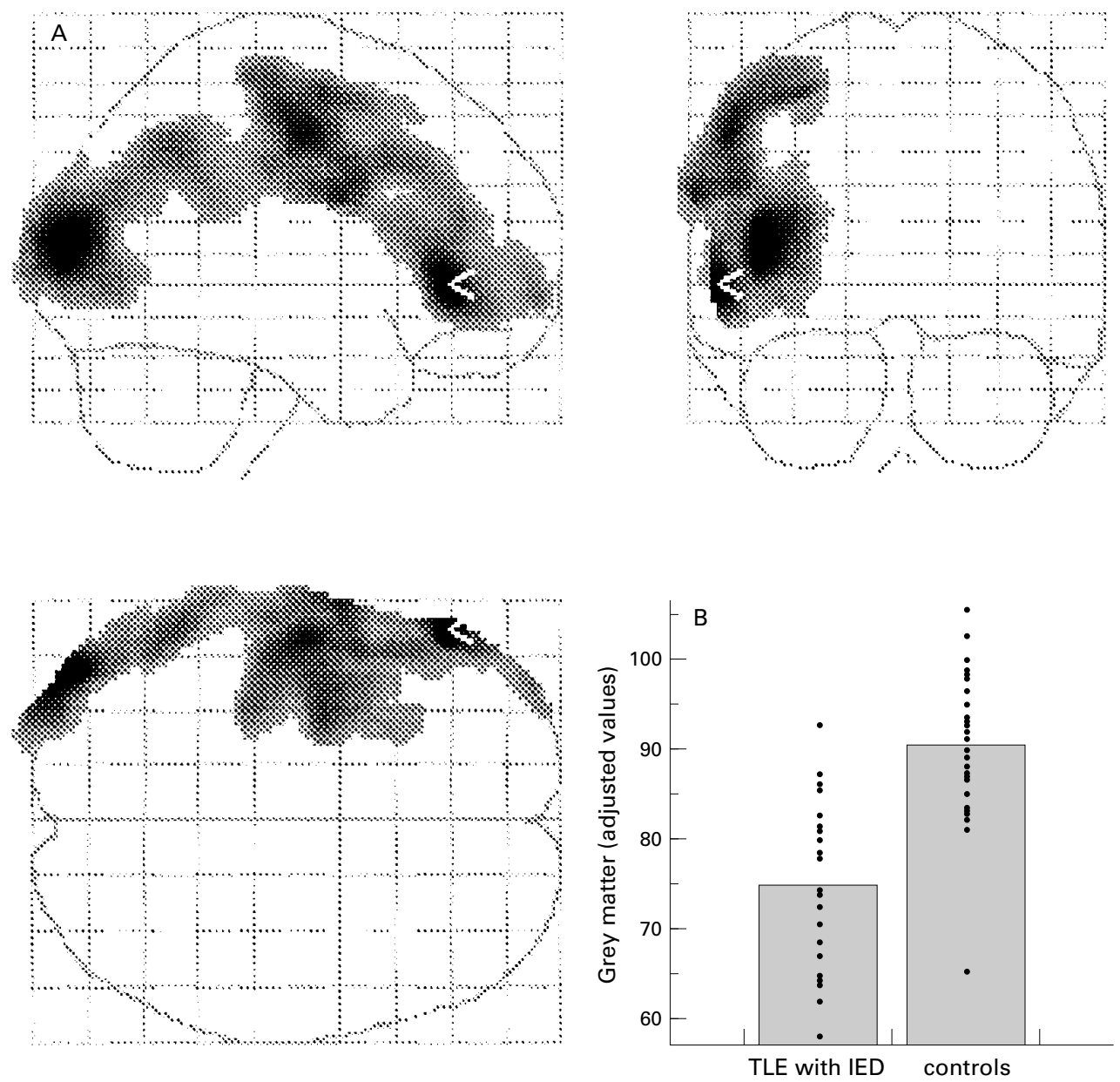

Figure 1 (A) "Glass brain" view of decreased grey matter in 24 patients with TLE with episodes of affective aggression compared with 35 control subjects; displayed after correction for multiple comparisons. The area of maximum difference (Talairach coordinates: $x=-58, y=36, z=9 \mathrm{~mm}$ ) is marked with a white arrow. Another peak area of significantly decreased grey matter ( $Z$ score $=4.78$ ) was found at Talairach coordinates $x=-66, y=0, z=28$. (B) Plot of adjusted values of grey matter demonstrates the comparison between the groups in the area of maximum difference (white arrow in $A$ ).

The resulting significant differences through the 3D image space were displayed collapsed into three orthogonal planes ("glass brain") (fig 1 A). Regions of significant difference were overlaid on normalised T1 weighted images to facilitate correlation with anatomy (fig 2). The Talairach coordinates ${ }^{16}$ are given in $\mathrm{mm}$ describing the location of significant voxels; $x$ defining the lateral displacement of this voxel from the midline (left=negative), $y$ defining the anteroposterior position relative to the anterior commissure (posterior $=$ negative), and $\mathrm{z}$ defining the vertical position relative to the line connecting the anterior and posterior commissure (down=negative).

\section{Results}

Combining clinical, electrophysiological, and qualitative MRI information, 14 patients with IED were diagnosed as having left sided TLE, four right sided TLE, and in seven patients with TLE, the seizure onset could not be lateralised using this information (three patients with IED had electrophysiological or imaging signs of bilateral temporal lobe involvement and three had findings equivocal concerning the lateralisation of their TLE). Eleven patients without IED had left sided TLE, nine right sided TLE, and in five patients without IED, the TLE could not be lateralised. None of these TLE lateralisations were overrepresented in either the TLE group with or without IED. One patient from each group could not be scanned for the current study because of a vagus nerve stimulator in one and claustrophobia in the other patient, although previous imaging was available for visual analysis (table 2). Table 2 summarises the MRI diagnoses on visual assessment and shows that amygdala or hippocampal sclerosis in patients with TLE was not associated with interictal aggressive episodes ${ }^{5}$; it also shows that right sided hippocampal sclerosis was more frequent in patients with TLE without IED.

Voxel by voxel group comparisons of automatically segmented grey matter were made between 24 patients with TLE each with or without IED and 35 control subjects. In patients with TLE with IED compared as a group with healthy control subjects, reductions of grey matter were found over large areas of the left extratemporal neocortex with maxima in the left frontal neocortex; one maximum difference projection had a $\mathrm{Z}$ score of 5.67 at Talairach coordinates $x=-58, y=36, z=9 \mathrm{~mm}$ (left anterior frontolateral cortex), the other a $\mathrm{Z}$ 


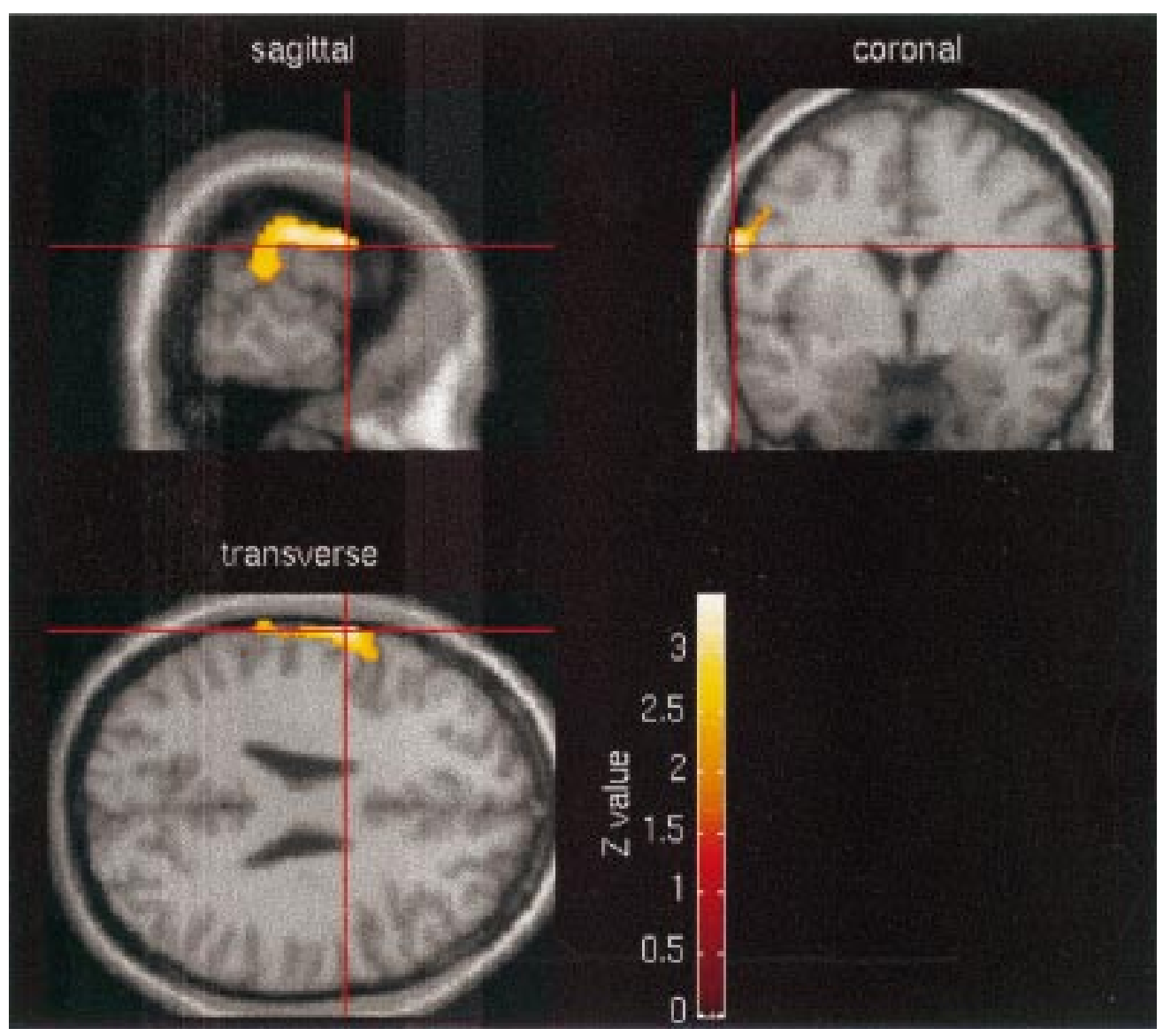

Figure 2 Area of decreased grey matter (overlaid on normalised T1 weighted MRI) comparing 24 patients with TLE with episodes of affective aggression with 24 patients with TLE without such episodes. At the location of maximum difference (Talairach coordinates: $x=-66, y=-2, z=26 \mathrm{~mm}$ ) the Zscore was 3.49 .

score of 4.78 in a more posterior left frontal lobe location (Talairach coordinates $\mathrm{x}=-66$, $\mathrm{y}=0, \mathrm{z}=28 \mathrm{~mm}$, fig $1 \mathrm{~A}$ and B). ${ }^{16}$ Patients with TLE who did not have IED showed no significant decrease of cortical grey matter compared with control subjects. Patients with TLE with IED also had reduction of left frontal grey matter, compared with patients with TLE without IED (fig 2), although less marked than when compared with control subjects ( $Z$ score $=3.49$ at Talairach coordinates $x=-66$, $y=-2, z=26 \mathrm{~mm}$ ). The SPM based voxel-wise correlation of SDAS scores and automatically segmented grey matter in all patients with TLE showed a left frontal grey matter area being negatively correlated with these scores which expressed social consequences of interictal affective aggression ( $\mathrm{Z}$ score 3.65 at Talairach coordinates $\mathrm{x}=-66, \mathrm{y}=-2, \mathrm{z}=26$ $\mathrm{mm})$. Age, scores of depression and anxiety, IQ measures, or scores of verbal fluency did not significantly correlate with specific decreases in grey matter in all patients with TLE.

In patients with TLE with IED compared with control subjects, increases of grey matter were found in the neocortex of the left temporal lobe, whereas similar changes were found bilaterally in patients with TLE without IED (fig $3 \mathrm{~A}$ and $\mathrm{B}$ ).

\section{Discussion}

This is the first MRI study of a possible structural correlate of affective aggression or IED in patients with epilepsy, using automated segmentation and voxel by voxel comparisons. We demonstrated a decrease in frontal grey matter in patients with TLE and IED compared with control subjects or with patients with TLE without IED. This finding adds localising information to prior knowledge of widespread, but subtle brain changes in patients with TLE and episodes of affective aggression. ${ }^{517}$

METHODOLOGICAL CONSIDERATION

Although multiple exogenous or endogenous factors may contribute to episodes of affective aggression, this paper focuses on the possible underlying structural substrate of this interictal clinical problem in patients with TLE.

The term "episodic dyscontrol" is controversial. There is no such condition included into the ICD- $10^{18}$ and there is still some discussion as to whether or not this dyscontrol syndrome exists as an independent entity. ${ }^{17}$ The diagnosis of "intermittent explosive disorder" has been included into the DSM-IV chapter on impulsecontrol disorders (DSM-IV 312.34). ${ }^{7}$ The diagnostic features of IED are basically those of episodic dyscontrol. ${ }^{3}{ }^{19}$ Using the DSM-IV criteria of IED operationally, we grouped patients 

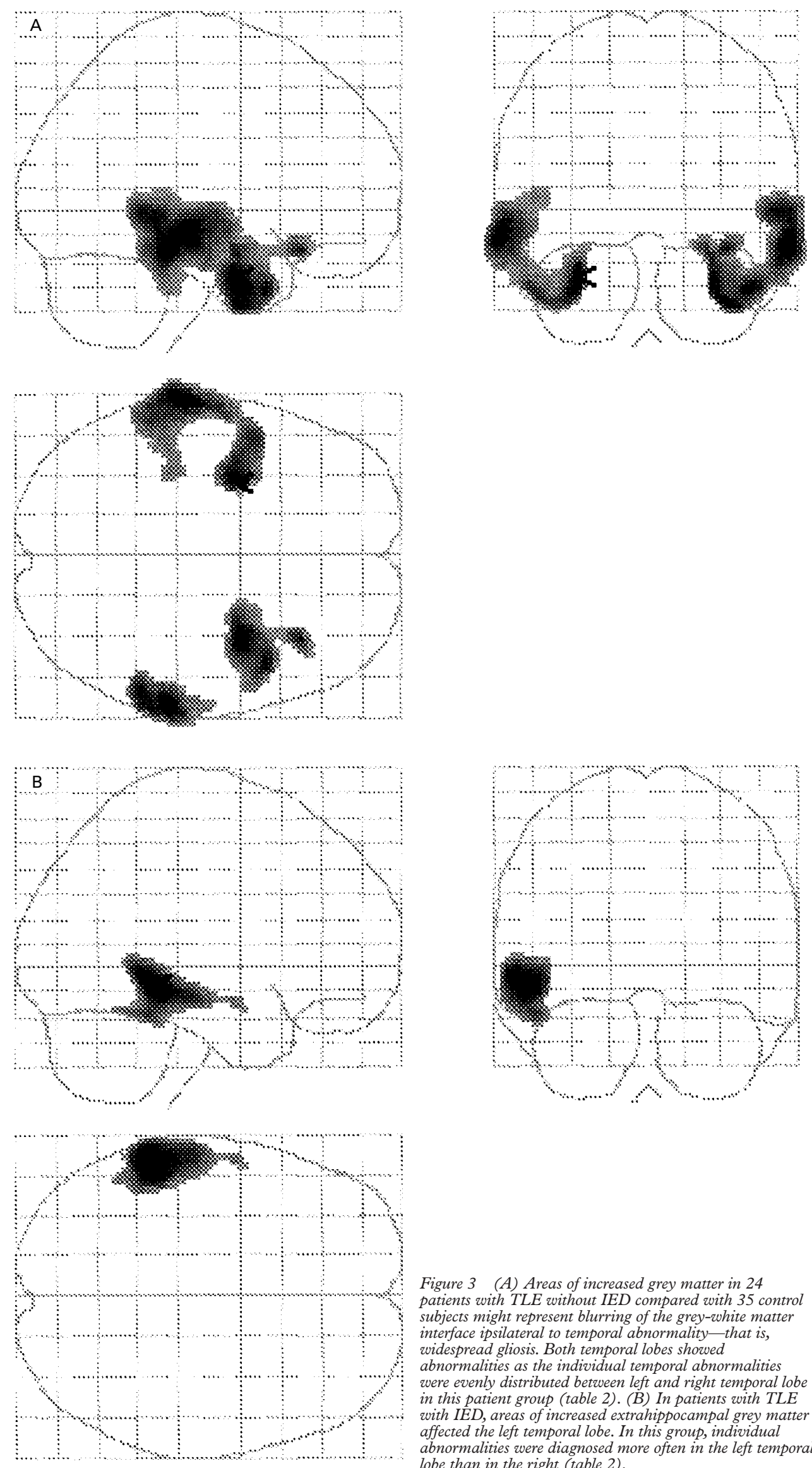

Figure 3 (A) Areas of increased grey matter in 24 patients with TLE without IED compared with 35 control subjects might represent blurring of the grey-white matter interface ipsilateral to temporal abnormality - that is, widespread gliosis. Both temporal lobes showed abnormalities as the individual temporal abnormalities were evenly distributed between left and right temporal lobe in this patient group (table 2). (B) In patients with TLE with IED, areas of increased extrahippocampal grey matter affected the left temporal lobe. In this group, individual abnormalities were diagnosed more often in the left temporal lobe than in the right (table 2). 
with TLE into those with IED and those without. Addressing the measurable social implications of these episodes of aggression, this classification was confirmed subsequently by subscores of validated questionnaires filled in by the next of kin or carers. ${ }^{5}$ However, the lack of a patient control group with IED but without TLE reduces the generalisbility of our finding of abnormal frontal neocortex beyond our study group.

There have been several studies assessing subtle brain abnormalities in patients with other neuropsychiatric disorders-for example, MRI based quantification using volume of interest approaches in depression and schizophrenia. ${ }^{2021}$ Voxel by voxel comparisons of neocortical grey matter and sometimes subcortical white matter, which were segmented automatically, have been used in patients with schizophrenia, depression, ${ }^{22-24}$ and Kallmann's syndrome. ${ }^{25}$ In patients with epilepsy, this voxel based method has been validated using semiautomated and automated segmentation and added localising information to the finding of widespread structural abnormalities demonstrated with a VOI based approach in patients with epilepsy. ${ }^{6}{ }^{26}$

In the current study, $\mathrm{T} 1$ weighted images were spatially normalised, automatically segmented, and smoothed before statistical tests were applied to the images on a voxel by voxel basis. The smoothed grey matter images can be thought of as representing the local amount of grey matter. ${ }^{22-26}$ The left frontal reduction of grey matter in patients with TLE and IED might be the imaging correlate of a localised decrease in grey matter volume or neuronal density. Extratemporal grey matter atrophy or neuronal loss in subgroups of patients with TLE, however, are still awaiting pathological confirmation.

Hippocampal sclerosis is common in patients with TLE ${ }^{27}$ but not linked to IED. ${ }^{5}$ We have shown recently ipsilateral temporal extrahippocampal, but not extratemporal abnormalities to be specifically associated with hippocampal sclerosis. ${ }^{6}$ Here, the same group finding, an increase of grey matter in the temporal neocortex, was demonstrated for both temporal lobes in patients with TLE without IED, possibly reflecting an even distribution of left and right sided hippocampal sclerosis throughout this group. In patients with TLE with IED, there was an increase of grey matter in the left extrahippocampal temporal neocortex, representing a ratio of left to right sided hippocampal sclerosis of $8: 1$ in this group (table 2). The aetiology of this group finding, a relative increase in temporal neocortical grey matter located at the inside of the automatically segmented grey matter, is unclear. It may represent diminished grey-white matter demarcation in the temporal lobe previously described on T2 weighted images in patients with hippocampal sclerosis and may be the imaging correlate of histological abnormalities including subpial gliosis, subcortical gliosis, microdysgenesis, or differences in cell densities detected in the neocortex of patients with intractable TLE. ${ }^{28}$
Problems in the diagnosis of epilepsy, inadequate sampling procedures, small sample sizes, lack of adequate control groups, inadequate measures of behavioural phenomena, and confounding variables such as intelligence have complicated previous studies of psychiatric abnormalities in patients with epilepsy. ${ }^{29}$ Most of these factors have been accounted for in this study. Confounding factors for an MRI study of cerebral grey matter such as age or sex distribution were controlled for by patient selection. There was, however, a difference in the IQ and verbal fluency between patients with TLE with or without IED (table 1). SPM analysis correlating the local amount of grey matter with IQ measures or verbal fluency, however, did not disclose specific changes in grey matter correlated over the range of these neuropsychological measures describing the patient population of this study. Previously, hyperarousal and dyscontrol, both phenomenological aspects of IED, have been associated with low intelligence and with low verbal abilities and were seen as results of subtle diffuse brain damage. $^{3031}$

\section{BIOLOGICAL IMPLICATIONS}

After comparison of automated segmented grey matter on a voxel by voxel basis, patients with TLE with IED had more structural abnormalities outside the affected temporal lobes than patients with TLE without IED. In this study, the reduction of neocortical grey matter was most marked in the left frontal grey matter in patients with TLE and IED, in addition to abnormalities detected on visual MRI assessment which showed a bias towards the left temporal lobe in this patient group (table 2). ${ }^{5}$ Previously, extratemporal neocortical changes in epilepsy not visible on high resolution MRI, but demonstrated with semiautomated segmentation and volume of interest based quantification have been correlated with more widespread neuropsychological abnormalities $^{32}$ and also with a poorer prognosis after temporal lobectomy in patients with TLE. ${ }^{33}$ Whether in patients with TLE, a history of interictal episodes of affective aggression might imply extratemporal abnormalities and leaves these patients less likely to be rendered seizure free by epilepsy surgery needs investigation.

The finding of a decrease in left frontal grey matter in patients with TLE with IED is relevant for understanding the neuronal circuitry related to aggression. In the past it has been suggested that abnormalities in cerebral structures which are involved in impulse control and stimulus recognition can lead to sudden outbursts of violent behaviour. ${ }^{17}$ Bear suggested that right sided lesions in the so called ventral system (consisting of inferior temporal cortex, temporolimbic structures, orbitofrontal cortex) could produce a transient release of aggressive responses in animals as long as there was no lesion to the right sided so called dorsal system (inferior parietal, cingulate, dorsofrontal cortex). ${ }^{34}$ These ideas have been controversial as many studies support a 
more important role of the left hemisphere in aggression. ${ }^{435} 36$

The reduced frontal grey matter we have shown might be a structural correlate with earlier findings from functional imaging and MR spectroscopy showing a reduced prefrontal glucose metabolism in murderers and significantly lower neuronal markers in the frontal lobes of repetitively violent patients with learning disabilities, although without clear lateralising effects. ${ }^{37}{ }^{38}$ These findings might suggest a localised reduction in frontal grey matter volume or neuronal density to be common to different syndromes involving dyscontrol or affective aggression.

The amygdala are critical structures within the cortical-subcortical circuits mediating aggressive behaviour, mainly implicated in processing emotional information and in hyperarousal. In animal models, stimulation of the amygdala leads to aggression whereas bilateral lesions render an animal tame. Further frontal stimulation inhibits the amygdala released aggression. ${ }^{39}$ Thus, at least part of the circuitry of aggression involves the interconnected amygdala and frontal cortices. In our patient groups, the frontal abnormalities might be correlated with dyscontrol, a failure of inhibition of aggressive behaviour. Bouts of aggression have been described as part of the frontal lobe syndromes both in the orbitofrontal (disinhibited) and frontal convexity (apathetic) syndromes. ${ }^{39}$ Although patients with TLE and IED scored lower on a test of frontal function (verbal fluency; table $1)^{40}$ than patients with TLE only, this difference was not correlated with any change in grey matter. Interactions between different brain regions involved in aggressive behaviour and associated additional neuropsychological impairment will have to be clarified in future studies. Voxel by voxel based studies such as ours might guide these future studies by providing results without the bias of operator dependent approaches.

\section{Conclusion}

This study suggests a connection between episodes of IED in patients with TLE and reduced frontal grey matter. It is, however, difficult to infer a causal relation from cross sectional data, even if our findings resulted from comparisons with a normal and a disease control group. A longitudinal quantitative MRI study in patients with TLE eventually developing episodes of interictal IED would be needed to distinguish aetiological from epilepsy or treatment related abnormalities. The technique used here is widely available, easy to implement, and could be readily applied to other patient groups.

We are grateful to Drs J Stevens and B Kendall for expert neuroradiological assessment of the original MRI, and J Ashburner, Functional Imaging Laboratory, Wellcome Department of Cognitive Neurology, Institute of Neurology, Queen Square, nitive Neurology, Institute of Neurology, Queen Square,
London, and Dr M Glabus, MRC Brain Metabolism Unit, London, and Dr M Glabus, MRC Brain Metabolism Unit, Royal Edinburgh Hospital, Edinburgh, UK, for their help with the implementation of SPM. We also thank The National Society for Epilepsy, Chalfont St Peter, UK (FGW, SLF, MJK, PJT, JSD), the Raymond Way Fund, Institute of Neurology, UCL, Gesellschaft, Albert-Ludwigs-Universität, Freiburg, Germany Gesellschaft, Albert-Ludwige
(LTVE) for their support.
1 Pritchard III PB, Lombroso CT, McIntyre M. Psychological complications of temporal lobe epilepsy. Neurology 1980;30:227-32.

2 Bach-Y-Rita G, Lion JR, Climent CE, et al. Episodic dyscontrol: a study of 130 violent patients. Am F Psychiatry 1971;127:1473-8.

3 Elliott FA. Neurological findings in adult minimal brain dysfunction and the dyscontrol syndrome. F Nerv Ment Dis 1982;170:680-7.

4 Saver JL, Salloway SP, Devinsky O, et al. Neuropsychiatry of aggression. In: BS Fogel, RB Schiffer, SM Rao, eds. Neuropsychiatry. Baltimore: Williams and Wilkins, 1996: 523-48.

5 Tebartz van Elst L, Woermann FG, Lemieux L, et al. Affective aggression in patients with temporal lobe epilepsy: a quantitative magnetic resonance imaging study of the amyquantitative magnetic resonance
gdala. Brain 1999 (in press).

6 Woermann FG, Free SL, Koepp MJ, et al. Voxel-by-voxel Woermann FG, Free SL, Koepp MJ, et al. Voxel-by-voxel
comparison of automatically segmented cerebral grey matter: a rater-independent comparison of structural MRI in patients with epilepsy. Neuroimage 1999;10:373-84.

7 American Psychiatric Association. Diagnostic and statistical manual of mental disorders - DSM-IV. Washington, DC: APA, 1994.

8 European Rating Aggression Group. Social dysfunction and aggression scale (SDAS-21) in generalised aggression and in aggressive attacks: a validity and reliability study. International fournal of Methods of Psychiatric Research 1992;2:15-29.

9 Mak M, De Koning P. Clinical research in aggressive patients: pitfalls in study design and measurement of gression. Prog Neuropsychopharmacol Biol Psychiatry 1995;19:993-1017.

10 Robb RA, Hanson DP, Karwoski RA, et al. Analyze: a comprehensive operator-interactive software package for multiprehensive operator-interactive software package for multiMed Imaging Graph 1989;13:433-54.

11 Friston KJ, Ashburner J, Frith CD, et al. Spatial registration and normalization of images. Human Brain Mapping 1995; $2: 165-89$

12 Friston KJ, Holmes AP, Worsley KJ, et al. Statistical parametric maps in functional imaging: a general linear approach. Human Brain Mapping 1995;2:189-210.

3 Ashburner J, Friston KJ. Multimodal image coregistration and partitioning: a unified framework. Neuroimage 1997;6: 209-17

14 Friston KJ, Frith CD, Liddle PF, et al. The relationship between global and local changes in PET scans. 7 Cereb Blood Flow Metab 1990;10:458-66.

15 Friston KJ, Worsley KJ, Frackowiak RSJ, et al. Assessing the significance of focal activations using their spatial extent. Human Brain Mapping 1994;1:210-20.

16 Talairach J, Tournoux P. A co-planar stereotactic atlas of the human brain. Stuttgart: Thieme, 1988.

17 Fenwick P. Episodic dyscontrol. In: Engel J, Pedley TA, eds. Epilepsy: a comprehensive textbook. Philadelphia: LippincottRaven, 1997:2767-74.

18 World Health Organisation. The ICD-10 classification of mental and behavioural disorders: clinical descriptions and diagnostic guidelines. Oxford: Oxford University Press, 1992.

19 Elliott FA. Violence: the neurologic contribution: an overview. Arch Neurol 1992;49:595-603.

20 Sheline YI, Wang PW, Gado MH, et al. Hippocampal atrophy in recurrent major depression. Proc Natl Acad Sci 1996;93:3908-13.

21 Lawrie SM, Abukmeil SS. Brain abnormality in schizophrenia. A systematic and quantitative review of volumetric nia. A systematic and quantitative review of volumetric 172:110-20.

22 Wright IC, McGuire PK, Poline JB, et al. A voxel-based method for the statistical analysis of gray and white matter density applied to schizophrenia. Neuroimage 1995;2:24452

23 Chua SE, Wright IC, Poline JB, et al. Grey matter correlates of syndromes in schizophrenia: a semi-automated analysis of structural magnetic resonance images. $\mathrm{Br} \mathcal{F}$ Psychiatry 1997;170:406-10.

24 Shah PJ, Ebmeier KP, Glabus MF, et al. Cortical grey matter reductions associated with treatment-resistant chronic unipolar depression. Br F Psychiatry 1998;172:527-32.

25 Krams M, Quinton R, Ashburner J, et al. Kallmann's syndrome: mirror movements associated with bilateral corticospinal tract hypertrophy. Neurology 1999;52:816-22.

26 Richardson MP, Friston KJ, Sisodiya SM, et al. Cortical grey matter and benzodiazepine receptors in malformations of cortical development: a voxel-based comparison of tructural and functional imaging data. Brain 1997;120: structural

27 Duncan JS. Imaging and epilepsy. Brain 1997;120:339-77.

28 Meiners LC, van Gils A, Jansen GH, et al. Temporal lobe epilepsy: the various MR appearances of histologically proven mesial temporal sclerosis. AfNR Am $\mathcal{f}$ Neuroradiol 1994;15:1547-55.

29 Levin R, Banks S, Berg B. Psychosocial dimensions of epilepsy: a review of the literature. Epilepsia 1988;29:80516.

30 Foster HG, Hillbrand M, Silverstein M. Neuropsychological deficit and aggressive behavior: a prospective study. Prog Neuropsychopharmacol Biol Psychiatry 1993;17:939-46.

31 Barratt ES, Stanford MS, Kent TA, et al. Neuropsychological and cognitive psychophysiological substrates of mpulsive agression. Biological Psychiatry 1997;41: 1045-61. 
32 Baxendale SA, Sisodiya SM, Thompson PJ, et al. Disproportion in the distribution of grey and white matter: neuropsychological correlates. Neurology 1999;52:248-52.

33 Sisodiya SM, Moran N, Free SL, et al. Correlation of widespread preoperative magnetic resonance imaging changes with unsuccessful surgery for hippocampal sclerosis. Ann Neurol 1997;41:490-6.

34 Bear DM. Hemispheric specialization and the neurology of emotion. Arch Neurol 1983;40:195-202

35 Nachson I. Neuropsychology of violent behavior: controversial issues and new developments in the study of hemispheric function. In: Miller JS, ed. Neuropsychology of aggression. Boston; Kluwer Academic, 1991:93-116.
36 Devinsky O, Ronsaville D, Cox C, et al. Interictal aggression in epilepsy: the buss-durkee hostility inventory. Epilepsia 1994;35:585-90.

37 Raine A, Stoddard J, Bihrle S, et al. Prefrontal glucose deficits in murderers lacking psychosocial deprivation. Neuropsychiatry Neuropsychology and Behavioural Neurology 1998;11:1-7.

38 Robertson D, Critchley H, Daly E, et al. The neurobiology of severe and repetitive violence: A H-1 MRS study of frontal lobe. Schizophr Res 1998;29:100.

39 Trimble MR. Biological psychiatry. Chichester: Wiley, 1996.

40 Crowe SF. Dissociation of two frontal lobe syndromes by a test of verbal fluency. F Clin Exp Neuropsychol 1992;14:32739.

\section{NEUROLOGICAL STAMP}

Ilya Metchinikoff (1845-1908)

The Nobel prize in 1908 was awarded jointly to llya Metchinikoff and Paul Ehrlich in recognition of their work on immunity. Metchinikoff was one of the most eminent of Pasteur's pupils. He was not medically qualified but taught zoology at Odessa and St Petersburg and from 1873-82 was Professor of Zoology and Comparative Anatomy at Odessa. He was an outspoken Darwinist and in the university's deteriorating political atmosphere at the time was labelled a "red". The Tsar's assassination in 1881 unleashed the reactionaries and antisemites. Metchinikoff tried suicide, on this occasion for the second time by injecting himself with relapsing fever germs. Despite cardiac damage he recovered.

Metchinikoff's epochal discoveries occurred in late 1882 in Messina, Sicily when during his investigations of digestive processes of larval starfish he noticed that after introduction of a foreign body or bacteria into the body of a transparent starfish, a large number of cells soon surrounded the foreign particle, finally absorbing and dissolving it. He compared the process to the accumulation of white blood cells in human inflammation. This led him to formulate the doctrine of phagocytosis, the destruction of bacteria by white blood cells. He invented the term phagocyte (Gr Phaegin, to eat) to connect defence with digestion. The pathologist Virchow encouraged him but warned that biomedical opinion generally would be hostile. Metchinikoff extended his theory by studying the role of phagocytosis in metamorphosis and then infectious disease. The fact that some infected animals succumbed while others survived raised the concept of immunity to infection.

In 1903 Metchinikoff succeeded with Emile Roux in transferring syphilis to apes and also made a preventive mercurous ointment. He also did research on cholera. His

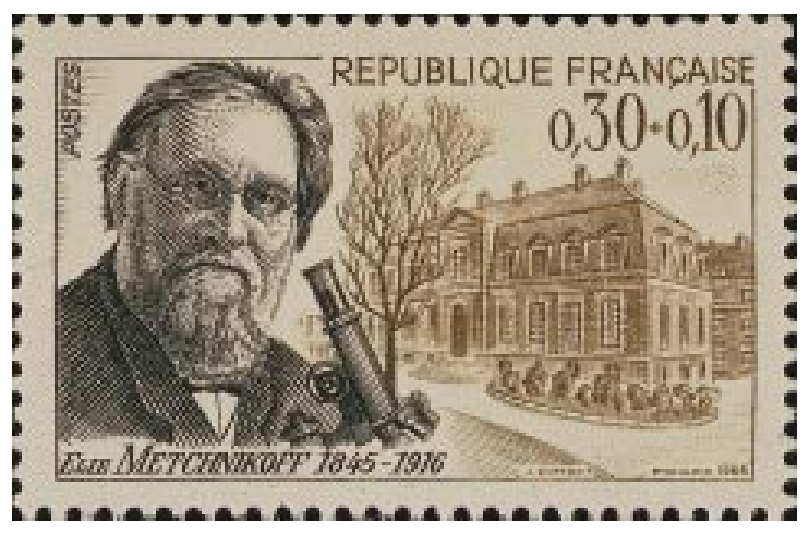

later years were largely concerned with the study of the aging factors in man. Methods of inducing longevity were discussed in The Nature of Man (1904) and The Prolongation of Human Life (1910). He devoted much attention to the question of intestinal sepsis and to the possibility of prolonging life by the ingestion of lactic acid bacilli. To study and control senescence Metchinikoff proposed the establishment of a new scientific discipline he named gerontology. In 1938 the first Congress of Aging was convened in Kiev and in 1962 the USSR Institute of Gerontology was founded. Conferences in the United States in the 1930s led to the formation of the Gerontology Society (1945) and eventually to the National Institute of Aging (1974).

An urn containing Metchinikoff's ashes was placed in the library of the Pasteur Institute. $\mathrm{He}$ has been philatelically honoured by Russia but is shown here with a microscope and the Pasteur Institute on a French stamp issued in 1966 (Stanley Gibbons 1707, Scott B398). 